\title{
Bifurcation of Nonlinear Conservation Laws from the Classical Energy Conservation Law for Internal Gravity Waves in Cylindrical Wave Field
}

\author{
N. H. Ibragimov ${ }^{1}$, R. N. Ibragimov ${ }^{2} *$ \\ ${ }^{1}$ Department of Mathematics and Science \\ Blekinge Institute of Technology, SE-371 79 Karlskrona, Sweden \\ ${ }^{2}$ Department of Mathematics \\ University of Texas at Brownsville, TX 78520, USA
}

\begin{abstract}
New conservation laws bifurcating from the classical form of conservation laws are constructed to the nonlinear Boussinesq model describing internal Kelvin waves propagating in a cylindrical wave field of an uniformly stratified water affected by the earth's rotation. The obtained conservation laws are different from the well known energy conservation law for internal waves and they are associated with symmetries of the Boussinesq model. Particularly, it is shown that application of Lie group analysis provide three infinite sets of nontrivial integral conservation laws depending on two arbitrary functions, namely $a(t, \theta), b(t, r)$ and an arbitrary function $c(t, \theta, r)$ which is given implicitly as a nontrivial solution of a partial differential equation involving $a(t, \theta)$ and $b(t, r)$.
\end{abstract}

Keywords and phrases: Kelvin internal waves, conservation laws

Mathematics Subject Classification: 76U05, 76B55, 35Q80

\section{Introduction}

A conservation law refers to an equation which relates the increase or decrease of a quantity to terms representing supply or destruction. In a continuous medium, the typical form of a conservation law is as follows:

$$
\frac{\partial P}{\partial t}+\operatorname{div} Q=S,
$$

in which $P$ is the quantity being "conserved", $Q$ is the "flux" (i.e. a transport of $P$ within the medium) and $S$ represents source $(S>0)$ or $\operatorname{sink}(S<0)$ terms. Application of the divergence theorem allows to write the conservation law (1.1) in an integral form as

$$
\frac{d}{d t} \int_{V} P d V=-\int_{\partial V} Q \cdot n d S+\int_{V} S d V .
$$

*Corresponding author. E-mail: Ranis. Ibragimov@utb.edu 
Invariably, conservation laws contain more terms than equations.

The emphasis in this paper will be on an application of Lie group analysis to derivation of conservation laws for the mathematical model describing nonlinear Kelvin waves in a cylindrical basin. Particulalrly, the classical energy conservation law for internal waves is a particular case of the form (1.1) or (1.2). In this work we will derive new conservation laws that "bifurcate" from the latter forms. The Kelvin wave is a large-scale wave motion of great practical importance in the Earth's atmosphere and ocean. The existence of the Kelvin wave relies on (i) a gravity and stable stratification for sustaining a gravitational oscillation, (ii) significant Coriolis acceleration, and (iii) the presence of vertical boundaries or the equator. Oceanic Kelvin waves play a critical role in tidal motion, in the adjustment of the tropical ocean to wind stress forcing, and in generating and sustaining the El Niño Southern Oscillaiton (e.g. the El Niño thermocline signal in the ocean propagates up to the west coast of North America as a Kelvin wave). There are two basic types of Kelvin waves: surface and internal Kelvin waves.

Internal costal Kelvin waves can be generated by wind-induced, time-dependent costal upwelling. Costal upwelling (downelling) is cased by an Ekman mass flux transported offshore (onshore) and forced by longshore winds. The disturbances can then propagate along the coast as boundary-trapped internal Kelvin waves (see also [14]). The internal Kelvin wave speed depends on the density difference across the interface and is normally much slower than that of surface Kelvin waves. In the ocean, the typical speed for internal costal Kelvin waves is of order of $1 \mathrm{~m} \mathrm{~s}^{-1}$ and the Rossby radius of deformation is of the order of $10 \mathrm{~km}$ in the latitudes.

In this paper, we address the equations of motion for internal waves affected by the earth's rotation. The inquiry is motivated by dynamically significant Coriolis forces in meteorology and oceanographic applications such as nonlinear climate variability models, the general atmospheric circulation model, weather prediction (see e.g., [3]; [18]) as well as a variety of applications to large-scale nonlinear dispersant operations e.g. Deepwater Horizon incident studied recently in [10].

The axes are $x$ (assumed eastward for definiteness) $y$ (northward) and $\widehat{k}$ is the unit vector with $z$ in the vertical direction (opposite gravity). The fluid velocity is $\vec{u}=(u, v, w)$ relative to the Cartesian coordinate system $(x, y, z)$. The hydrostatic approximation is assumed and the equations of motion are linearized about a background stable density profile $\bar{\rho}(z)$ with the associated buoyancy frequency. Motion is considered to be outside frictional boundary layers, allowing viscosity and diffusion to be neglected. In the theory of ocean circulation and within meteorological applications, it is commonplace to make the Boussinesq approximation in which the full variation of density is retained only in the buoyancy force in the vertical momentum balance. Wherever else it occurs, in the horizontal momentum balance, and in the continuity equation, density is replaced by a constant (in the simplest implementation). In particular, this means that the three-dimensional velocity field is assumed to be solenoidal (see e.g. [20]). Within the Boussinesq approximation, the governing equations of motion for internal waves, observed in a system of coordinates rotating with angular velocity $\vec{\Omega}$ are written as a non-hydrostatic analytical model in the form (see e.g., [8])

$$
\begin{aligned}
\rho_{0}\left[\frac{\partial \vec{u}}{\partial t}+\vec{u} \cdot \nabla \vec{u}+2 \vec{\Omega} \times \vec{u}\right] & =-\nabla p-g \rho \widehat{k}, \\
\frac{\partial \rho}{\partial t}+\vec{u} \cdot \nabla \rho+w \frac{d \bar{\rho}}{d z} & =0, \\
\nabla \cdot \vec{u} & =0,
\end{aligned}
$$

where $g$ is the acceleration due to gravity so that $p$ and $\rho$ are to be interpreted as the pressure and density departures from their mean state

$$
\bar{\rho}(z)=-\frac{\rho_{0}}{g} N^{2} z, \quad \bar{p}(z)=p_{0}-\rho_{0} g z-g \int_{0}^{z} \bar{\rho}(\xi) d \xi
$$


in which $\rho_{0}$ is the constant reference density, $N$ is the buoyancy frequency defined by

$$
N^{2}=-\frac{g}{\rho_{0}} \frac{d \bar{\rho}}{d z}
$$

and we require $\rho_{0}+\bar{\rho}$ and $\bar{p}$ to be consistent with the state of rest, i.e.,

$$
\frac{d \bar{p}}{d z}=-\left(\rho_{0}+\bar{\rho}\right) g
$$

The traditional $f$-plane approximation is made whereby we take $2 \vec{\Omega}=(0,0, f)$, where $f$ is the inertial frequency which depends on the rotation rate of the earth (angular velocity $\Omega=2 \pi \mathrm{rad} /$ day $\approx 0.73 \times 10^{-4}$ $\mathrm{s}^{-1}$ ). We derive the conservation laws for the nonlinear (1.3) - (1.5) in a cylindrical wave field including the effects of the earth's rotation and under the Kelvin's hypothesis saying that the component of the velocity that is normal to the lateral circular boundary be zero throughout the domain.

One can associate conservation laws with symmetries on any system of differential equations provided that the system under consideration is nonlinearly self-adjoint in the terminology of [11]. The system in question can be determined (the number of the equations in the system is equal to the number of dependent variables), over-determined (the number of the equations in the system is more than the number of dependent variables) or sub-definite (the number of the equations in the system is less than the number of dependent variables).

\section{Nonlinear cylindrical internal wave field}

Let there be a cylindrical model basin with radius $r_{0}$ and of the depth $H$ with $z \in[0, H]$. For completeness, we write explicitly the three-dimensional nonlinear Euler equations of motion (1.3) - (1.5) in the cylindrical domain within the Boussinesq approximation:

$$
\begin{aligned}
\frac{\partial u_{r}}{\partial t}+u_{r} \frac{\partial u_{r}}{\partial r}+\frac{u_{\theta}}{r} \frac{\partial u_{r}}{\partial \theta}+w \frac{\partial u_{r}}{\partial z}-\frac{u_{\theta}^{2}}{r}-f u_{\theta} & =-\frac{\partial p}{\partial r} \\
\frac{\partial u_{\theta}}{\partial t}+u_{r} \frac{\partial u_{\theta}}{\partial r}+\frac{u_{\theta}}{r} \frac{\partial u_{\theta}}{\partial \theta}+w \frac{\partial u_{\theta}}{\partial z}+\frac{u_{r} u_{\theta}}{r}+f u_{r} & =-\frac{1}{r} \frac{\partial p}{\partial \theta} \\
\frac{\partial w}{\partial t}+u_{r} \frac{\partial w}{\partial r}+\frac{u_{\theta}}{r} \frac{\partial w}{\partial \theta}+w \frac{\partial w}{\partial z} & =-\frac{\partial p}{\partial z}-\rho g \\
\frac{\partial \rho}{\partial t}+u_{r} \frac{\partial \rho}{\partial r}+\frac{u_{\theta}}{r} \frac{\partial \rho}{\partial \theta} & =\frac{N^{2}}{g} w \\
\frac{1}{r} \frac{\partial\left(r u_{r}\right)}{\partial r}+\frac{1}{r} \frac{\partial u_{\theta}}{\partial \theta}+\frac{\partial w}{\partial z} & =0
\end{aligned}
$$

As the particular example, we first write the classical energy conservation law associated with the linearized Boussinesq model (2.1) - (2.5), i.e.

$$
\begin{aligned}
\frac{\partial u_{r}}{\partial t}-f u_{\theta} & =-\frac{\partial p}{\partial r} \\
\frac{\partial u_{\theta}}{\partial t}+f u_{r} & =-\frac{1}{r} \frac{\partial p}{\partial \theta} \\
\frac{\partial w}{\partial t} & =-\frac{\partial p}{\partial z}-\rho g, \\
\frac{\partial \rho}{\partial t}-\frac{N^{2}}{g} w & =0 \\
\frac{1}{r} \frac{\partial\left(r u_{r}\right)}{\partial r}+\frac{1}{r} \frac{\partial u_{\theta}}{\partial \theta}+\frac{\partial w}{\partial z} & =0
\end{aligned}
$$


Multiplication of (2.6) by $u_{r},(2.7)$ by $u_{\theta}$ and (2.8) by $w$, and summing the result yileds the term

$$
\frac{\partial}{\partial t}\left[\frac{1}{2}\left(u_{r}^{2}+u_{\theta}^{2}+w^{2}\right)\right]+\rho g w+\operatorname{div}_{(r, \theta, z)}(p \vec{u})
$$

in which (2.10) has been used, the notation $\operatorname{div}_{(r, \theta, z)}$ means the divergence in cylindrical coordinates and $\vec{u}=\left(r u_{r}, u_{\theta}, w\right)$. The first two term in (2.11) are associated with the rates of change of kinetic and potential energy respectively whereas the last term represents the net work done by pressure forces. Using (2.9), we can rewrite the rate of change of the potential energy as

$$
\rho g w=\frac{g^{2}}{2 N^{2}} \frac{\partial}{\partial t} \rho^{2}
$$

and so the energy conservation law is written as

$$
\frac{\partial}{\partial t}\left[\frac{1}{2}\left(u_{r}^{2}+u_{\theta}^{2}+w^{2}+\frac{g^{2} \rho^{2}}{N^{2}}\right)\right]+\frac{1}{r} \frac{\partial}{\partial r}\left(r u_{r} p\right)+\frac{1}{r} \frac{\partial}{\partial \theta}\left(u_{\theta} p\right)+\frac{\partial}{\partial z}(w p)=0 .
$$

As he recounted in this presentation to the Royal Society of Edinburgh in 1879, Lord Kelvin thought that the vanishing of the velocity component normal to the wall suggested the possibility that it be zero everywhere. Following this hypothesis, we allow the component of the velocity that is normal to the lateral circular boundary be zero, i.e. we let, in anticipation,

$$
u_{r}=0
$$

throughout the domain and investigate the consequences.

As the next particular example, we investigate the effect of the Kelvin's hypothesis (2.14) on the classical energy conservation law (2.13) associated with the linearized Boussinesq model (2.6) - (2.10). Setting $u_{r}=0$ in $(2.6)-(2.10)$ and repeating the above procedure we recover the corresponding energy conservation law (2.13) under the Kelvin's hypothesis:

$$
\frac{\partial}{\partial t}\left[\frac{1}{2}\left(u_{\theta}^{2}+w^{2}+\frac{g^{2} \rho^{2}}{N^{2}}\right)\right]+\frac{1}{r} \frac{\partial}{\partial \theta}\left(u_{\theta} p\right)+\frac{\partial}{\partial z}(w p)-f u_{\theta}+\frac{\partial p}{\partial r}=0,
$$

in which

$$
-f u_{\theta}+\frac{\partial p}{\partial r}=0
$$

due to $(2.6)$.

While the most of the previous studies of Kelvin waves are based on linear modeling, the problem which forms the main focus of interest here is to obtain the conservation laws of the nonlinear Boussinesq model (2.1) - (2.5) within Kelvin's hypothesis (2.14):

$$
\begin{aligned}
& F_{1} \equiv \frac{\partial u_{\theta}}{\partial t}+\frac{u_{\theta}}{r} \frac{\partial u_{\theta}}{\partial \theta}+w \frac{\partial u_{\theta}}{\partial z}+\frac{1}{r} \frac{\partial p}{\partial \theta}=0, \\
& F_{2} \equiv \frac{\partial w}{\partial t}+\frac{u_{\theta}}{r} \frac{\partial w}{\partial \theta}+w \frac{\partial w}{\partial z}+\frac{\partial p}{\partial z}+\rho g=0, \\
& F_{3} \equiv \frac{\partial p}{\partial r}-\frac{u_{\theta}^{2}}{r}-f u_{\theta}=0 \\
& F_{4} \equiv \frac{\partial \rho}{\partial t}+\frac{u_{\theta}}{r} \frac{\partial \rho}{\partial \theta}-\frac{N^{2}}{g} w=0, \\
& F_{5} \equiv \frac{1}{r} \frac{\partial u_{\theta}}{\partial \theta}+\frac{\partial w}{\partial z}=0 .
\end{aligned}
$$


Our model is idealized by assuming $N$ to be uniform over the extent of the fluid. This corresponds to a vertically linear density variation. While this simplification is commonly used in laboratory and theoretical studies and it is quite reasonable for the thermocline region, it is not common in the deep region of the ocean with the except when considering short wavelengths in comparisons with the scale of density changes [19]. At low frequencies, close to $f$, rotational effects are important. Such internal waves are sometimes called inertial-internal waves. At high frequencies, close to $N$ and far from $f$, rotational effects are negligible.

\section{Symmetries and self-adjointness}

The symmetries of Eqs. (2.16) - (2.20) contain two arbitrary functions, $\varphi(z), \quad \psi(t)$, and are spanned by the following operators:

$$
\begin{aligned}
& X_{1}=\frac{\partial}{\partial t}, \quad X_{2}=\frac{\partial}{\partial \theta}, \quad X_{3}=\frac{\partial}{\partial z}, \quad X_{\varphi}=g \varphi(z) \frac{\partial}{\partial p}-\varphi^{\prime}(z) \frac{\partial}{\partial \rho}, \quad X_{\psi}=\psi(t) \frac{\partial}{\partial p} \\
& X_{4}=r \frac{\partial}{\partial r}+z \frac{\partial}{\partial z}+u_{\theta} \frac{\partial}{\partial u_{\theta}}+w \frac{\partial}{\partial w}+2 p \frac{\partial}{\partial p}+\rho \frac{\partial}{\partial \rho}, \\
& X_{5}=2(f t+2 \theta) \frac{\partial}{\partial \theta}-4 r \frac{\partial}{\partial r}+2 f r \frac{\partial}{\partial u_{\theta}}+f^{2} r^{2} \frac{\partial}{\partial p} .
\end{aligned}
$$

There are no group extensions in the case of the homogeneous fluid $(N=0)$ and in the following physically irrelevant case for the ocean $f=N$. For example, in the mid/high latitude upper non-homogeneous ocean $N$ is typically one or two orders of magnitude larger than $f$ ( $N$ varies from $10^{-3}$ to $10^{-2} \mathrm{~s}^{-1}$ while $f \sim 10^{-4} \mathrm{~s}^{-1}$ ). In agreement with [4] and [2], the presence of the arbitrary functions $\varphi(z), \psi(t)$ in the symmetry Lie algebra (3.1) is a characteristic property of incompressible fluids. For example,the operator $X_{\psi}$ generates the group transformation $\bar{p}=p+\varepsilon_{\psi} \psi(t)$ of the pressure $p$, where $\varepsilon_{\psi}$ is the group parameter associated with the latter particular symmetry. The invariance of fluid flows under this transformation is quite obvious because the velocity vector $\left(u_{\theta}, w\right)$ is invariant under this transformation.

In terms of group theoretical modeling, the dynamics of the system in the question can often be described by means of the formal Lagrangian and the adjoint equation. We will first show that the system of equations (2.16)-(2.16) is nonlinearly self-adjoint in the terminology of [11], [12]. To this end, we prove the following

Proposition. The over-determined system of equations (2.16)-(2.20) is nonlinearly self-adjoint.

Proof. The formal Lagrangian $\mathcal{L}$ for the system $(2.16)-(2.20)$ is:

$$
\begin{aligned}
& \mathcal{L}=U\left(\frac{\partial u_{\theta}}{\partial t}+\frac{u_{\theta}}{r} \frac{\partial u_{\theta}}{\partial \theta}+w \frac{\partial u_{\theta}}{\partial z}\right)+\frac{1}{r} \frac{\partial p}{\partial \theta}+V\left(\frac{\partial w}{\partial t}+\frac{u_{\theta}}{r} \frac{\partial w}{\partial \theta}+w \frac{\partial w}{\partial z}+\frac{\partial p}{\partial z}+\rho g\right) \\
& +P\left(\frac{\partial p}{\partial r}-\frac{u_{\theta}^{2}}{r}-f u_{\theta}\right)+R\left(\frac{\partial \rho}{\partial t}+\frac{u_{\theta}}{r} \frac{\partial \rho}{\partial \theta}-\frac{N^{2}}{g} w\right)+Q\left(\frac{1}{r} \frac{\partial u_{\theta}}{\partial \theta}+\frac{\partial w}{\partial z}\right),
\end{aligned}
$$

where $U, V, P, R$ and $Q$ are new dependent variables.

The adjoint system to Eqs. (2.16) - (2.20) is written

$$
F_{1}^{*} \equiv \frac{\delta \mathcal{L}}{\delta u_{\theta}}=0, \quad F_{2}^{*} \equiv \frac{\delta \mathcal{L}}{\delta w}=0, \quad F_{3}^{*} \equiv \frac{\delta \mathcal{L}}{\delta p}=0, \quad F_{4}^{*} \equiv \frac{\delta \mathcal{L}}{\delta \rho}=0
$$


Using the formal Lagrangian (3.2) we obtain:

$$
\begin{aligned}
& \frac{\delta \mathcal{L}}{\delta u_{\theta}}=-\frac{\partial U}{\partial t}-\frac{u_{\theta}}{r} \frac{\partial U}{\partial \theta}-w \frac{\partial U}{\partial z}-U \frac{\partial w}{\partial z}+\frac{V}{r} \frac{\partial w}{\partial \theta}+\frac{R}{r} \frac{\partial \rho}{\partial \theta}-\frac{2 u_{\theta}}{r} P-\frac{1}{r} \frac{\partial Q}{\partial \theta}-f P, \\
& \frac{\delta \mathcal{L}}{\delta w}=-\frac{\partial V}{\partial t}-\frac{u_{\theta}}{r} \frac{\partial V}{\partial \theta}-\frac{V}{r} \frac{\partial u_{\theta}}{\partial \theta}-w \frac{\partial V}{\partial z}-\frac{\partial Q}{\partial z}+U \frac{\partial u_{\theta}}{\partial z}-\frac{N^{2}}{g} R, \\
& \frac{\delta \mathcal{L}}{\delta p}=-\frac{\partial P}{\partial r}-\frac{1}{r} \frac{\partial U}{\partial \theta}-\frac{\partial V}{\partial z}, \quad \frac{\delta \mathcal{L}}{\delta \rho}=-\frac{\partial R}{\partial t}-\frac{u_{\theta}}{r} \frac{\partial R}{\partial \theta}-\frac{R}{r} \frac{\partial u_{\theta}}{\partial \theta}+g V .
\end{aligned}
$$

According to [11] the system (2.16) - (2.20) is nonlinearly self-adjoint if there exists a substitution

$$
U=\varphi^{1}, \quad V=\varphi^{2}, \quad P=\varphi^{3}, \quad R=\varphi^{4}, \quad Q=\varphi^{5},
$$

where $\varphi^{1}, \ldots, \varphi^{5}$ are functions of $t, \theta, r, z, u, w, p, \rho$ not vanishing simultaneously, such that the following equations are satisfied (Eqs. (3.5) from [11]):

$$
\left.F_{\alpha}^{*}\right|_{(3.5)}=\lambda_{\alpha}^{\bar{\beta}} F_{\bar{\beta}}, \quad \alpha=1, \ldots, 4 .
$$

Here $F_{\bar{\beta}}(\bar{\beta}=1, \ldots, 5)$ and $F_{\alpha}^{*}(\alpha=1, \ldots, 4)$ are given by the equations $(2.16)-(2.20)$ and $(3.4)$, respectively, $\lambda_{\alpha}^{\bar{\beta}}$ are undetermined coefficients, the symbol $\left.\right|_{(3.5)}$ indicates that the non-physical variables $U, \ldots, Q$ and their derivatives are eliminated by means of the substitution (3.5). The derivatives are computed in a usual way, e.g.

$$
\begin{aligned}
& \frac{\partial U}{\partial t}=D_{t}\left(\varphi^{1}\right) \equiv \frac{\partial \varphi^{1}}{\partial t}+\frac{\partial \varphi^{1}}{\partial u_{\theta}} \frac{\partial u_{\theta}}{\partial t}+\frac{\partial \varphi^{1}}{\partial w} \frac{\partial w}{\partial t}+\frac{\partial \varphi^{1}}{\partial p} \frac{\partial p}{\partial t}+\frac{\partial \varphi^{1}}{\partial \rho} \frac{\partial \rho}{\partial t} \\
& \frac{\partial U}{\partial \theta}=D_{\theta}\left(\varphi^{1}\right) \equiv \frac{\partial \varphi^{1}}{\partial \theta}+\frac{\partial \varphi^{1}}{\partial u_{\theta}} \frac{\partial u_{\theta}}{\partial \theta}+\frac{\partial \varphi^{1}}{\partial w} \frac{\partial w}{\partial \theta}+\frac{\partial \varphi^{1}}{\partial p} \frac{\partial p}{\partial \theta}+\frac{\partial \varphi^{1}}{\partial \rho} \frac{\partial \rho}{\partial \theta} \\
& \frac{\partial U}{\partial z}=D_{z}\left(\varphi^{1}\right) \equiv \frac{\partial \varphi^{1}}{\partial z}+\frac{\partial \varphi^{1}}{\partial u_{\theta}} \frac{\partial u_{\theta}}{\partial z}+\frac{\partial \varphi^{1}}{\partial w} \frac{\partial w}{\partial z}+\frac{\partial \varphi^{1}}{\partial p} \frac{\partial p}{\partial z}+\frac{\partial \varphi^{1}}{\partial \rho} \frac{\partial \rho}{\partial z} \\
& \frac{\partial Q}{\partial \theta}=D_{\theta}\left(\varphi^{5}\right) \equiv \frac{\partial \varphi^{5}}{\partial \theta}+\frac{\partial \varphi^{5}}{\partial u_{\theta}} \frac{\partial u_{\theta}}{\partial \theta}+\frac{\partial \varphi^{5}}{\partial w} \frac{\partial w}{\partial \theta}+\frac{\partial \varphi^{5}}{\partial p} \frac{\partial p}{\partial \theta}+\frac{\partial \varphi^{5}}{\partial \rho} \frac{\partial \rho}{\partial \theta}
\end{aligned}
$$

where

$$
\varphi^{1}=\varphi^{1}\left(t, \theta, r, z, u_{\theta}, w, p, \rho\right) \quad \text { and } \quad \varphi^{5}=\varphi^{5}\left(t, \theta, r, z, u_{\theta}, w, p, \rho\right) .
$$

The coefficients $\lambda_{\alpha}^{\bar{\beta}}$ and the functions $\varphi^{1}, \ldots, \varphi^{5}$ are found by solving four equations (3.6) corresponding to $\alpha=1, \ldots, 4$. The first equation corresponding to $\alpha=1$ is written

$$
F_{1}^{*}=\lambda_{1}^{1} F_{1}+\lambda_{1}^{2} F_{2}+\lambda_{1}^{3} F_{3}+\lambda_{1}^{4} F_{4}+\lambda_{1}^{5} F_{5} .
$$

We substitute in $F_{1}^{*}$ given by the first equation (3.4) the expressions (3.7) for $U_{t}, \ldots, Q_{\theta}$, equate the coefficients for

in both sides of Eq. (3.8) and obtain:

$$
\frac{\partial u_{\theta}}{\partial t}, \quad \frac{\partial w}{\partial t}, \quad \frac{\partial p}{\partial t}, \quad \frac{\partial \rho}{\partial t}
$$

$$
\lambda_{1}^{1}=-\frac{\partial \varphi^{1}}{\partial u_{\theta}}, \quad \lambda_{1}^{2}=-\frac{\partial \varphi^{1}}{\partial w}, \quad \varphi_{p}^{1}=0, \quad \lambda_{1}^{4}=-\frac{\partial \varphi^{1}}{\partial \rho} .
$$

Likewise, considering the c coefficients for

$$
\frac{\partial p}{\partial r}, \quad \frac{\partial p}{\partial z}, \quad \frac{\partial \rho}{\partial z}
$$


we obtain:

$$
\lambda_{1}^{3}=0, \quad \lambda_{1}^{2}=0, \quad \frac{\partial \varphi^{1}}{\partial \rho}=0 .
$$

Summarizing the equations (3.9) and (3.10) we see that

$$
\varphi^{1}=\varphi^{1}\left(t, \theta, r, z, u_{\theta}\right)
$$

and that

$$
\lambda_{1}^{1}=-\frac{\partial \varphi^{1}}{\partial u_{\theta}}, \quad \lambda_{1}^{2}=\lambda_{1}^{3}=\lambda_{1}^{4}=0 .
$$

In view of (3.12) Eq. (3.8) becomes

$$
F_{1}^{*}=-\frac{\partial \varphi^{1}}{\partial u_{\theta}} F_{1}+\lambda_{1}^{5} F_{5}
$$

We continue the similar calculations with Eq. (3.13) and with the remaining three equations (3.6) corresponding to $\alpha=2,3,4$. After lengthy but regular calculations we conclude that the substitution (3.5) satisfying Eqs. (3.6) has the following form:

$$
\begin{aligned}
& U=a(t, \theta) r+b(t, r), \quad V=0, \\
& P=-\frac{\partial a(t, \theta)}{\partial \theta} r, \quad R=0, \\
& Q=[a(t, \theta) r+b(t, r)] u_{\theta}+c(t, \theta, r),
\end{aligned}
$$

where $a(t, \theta), b(t, r)$ are arbitrary functions, and $c(t, \theta, r)$ is determined by the differential equation

$$
\frac{1}{r} \frac{\partial c}{\partial \theta}=f r \frac{\partial a(t, \theta)}{\partial \theta}-\left[r \frac{\partial a(t, \theta)}{\partial t}+\frac{\partial b(t, r)}{\partial t}\right] .
$$

Substituting (3.14) in (3.4) one can verify that the nonlinear self-adjointness conditions (3.6) of Eqs. (2.16) - (2.20) are satisfied in the following form:

$$
F_{1}^{*}=-[a(t, \theta) r+b(t, r)] F_{5}, \quad F_{2}^{*}=0, \quad F_{3}^{*}=0, \quad F_{4}^{*}=0 .
$$

Thus the system (2.16) - (2.20) is nonlinearly self-adjoint.

\section{Nonlinear conservation laws}

Conserved vectors associated with symmetries

$$
X=\xi^{i} \frac{\partial}{\partial x^{i}}+\eta^{\alpha} \frac{\partial}{\partial u^{\alpha}}
$$

of any nonlinearly self-adjoint system of differential equations

$$
F_{\bar{\alpha}}\left(x, u, u_{(1)}, \ldots, u_{(s)}\right)=0, \quad \bar{\alpha}=1, \ldots, \bar{m},
$$

is given by the following formula (see [11]), Eq. (8.23)):

$$
\begin{aligned}
& C^{i}=W^{\alpha}\left[\frac{\partial \mathcal{L}}{\partial u_{i}^{\alpha}}-D_{j}\left(\frac{\partial \mathcal{L}}{\partial u_{i j}^{\alpha}}\right)+D_{j} D_{k}\left(\frac{\partial \mathcal{L}}{\partial u_{i j k}^{\alpha}}\right)-\ldots\right] \\
& +D_{j}\left(W^{\alpha}\right)\left[\frac{\partial \mathcal{L}}{\partial u_{i j}^{\alpha}}-D_{k}\left(\frac{\partial \mathcal{L}}{\partial u_{i j k}^{\alpha}}\right)+\ldots\right]+D_{j} D_{k}\left(W^{\alpha}\right)\left[\frac{\partial \mathcal{L}}{\partial u_{i j k}^{\alpha}}-\ldots\right] .
\end{aligned}
$$


Here $\mathcal{L}=v^{\bar{\beta}} F_{\bar{\beta}}$ is the formal Lagrangian for the system in question and

$$
W^{\alpha}=\eta^{\alpha}-\xi^{j} u_{j}^{\alpha}
$$

The "non-physical variables" $v^{\bar{\alpha}}$ should be eliminated from the vector (4.1) by using the substitution

$$
v^{\bar{\alpha}}=\varphi^{\bar{\alpha}}(x, u), \quad \bar{\alpha}=1, \ldots, \bar{m},
$$

connecting the adjoint system with the system under consideration ([11]), Section 8.2).

We will apply the formula (4.1) to our system (2.16) - (2.20) by using the notation

$$
\begin{aligned}
& x^{1}=t, \quad x^{2}=\theta, \quad x^{3}=r, \quad x^{4}=z, \\
& u^{1}=u, \quad u^{2}=w, \quad u^{3}=p, \quad u^{4}=\rho .
\end{aligned}
$$

According to the notation (4.2), the conservation laws will be written in the form

$$
\left[D_{t}\left(C^{1}\right)+D_{\theta}\left(C^{2}\right)+D_{r}\left(C^{3}\right)+D_{z}\left(C^{4}\right)\right]_{(2.16)-(2.20)}=0,
$$

where $D_{t}, \ldots, D_{z}$ denote the total differentiations in $t, \ldots, z$.

In the case of the first-order formal Lagrangian (3.2) the formula (4.1) is written

$$
C^{i}=W^{\alpha} \frac{\partial \mathcal{L}}{\partial u_{i}^{\alpha}} .
$$

Substituting in (4.4) the expression (3.2) of the formal Lagrangian by taking into account the notation (4.2) and the equation $V=R=0$ due to Eqs. (3.14) we obtain:

$$
\begin{aligned}
& C^{1} \equiv W^{\alpha} \frac{\partial \mathcal{L}}{\partial u_{t}^{\alpha}}=U W^{1}, \\
& C^{2} \equiv W^{\alpha} \frac{\partial \mathcal{L}}{\partial u_{\theta}^{\alpha}}=\frac{1}{r}(u U+Q) W^{1}+\frac{1}{r} U W^{3}, \\
& C^{3} \equiv W^{\alpha} \frac{\partial \mathcal{L}}{\partial u_{r}^{\alpha}}=P W^{3}, \\
& C^{4} \equiv W^{\alpha} \frac{\partial \mathcal{L}}{\partial u_{z}^{\alpha}}=w U W^{1}+Q W^{2} .
\end{aligned}
$$

We replace here $U, P$ and $Q$ with their values given in (3.14) and arrive at the following final formula for calculating the conserved vectors:

$$
\begin{aligned}
& C^{1}=(a r+b) W^{1}, \\
& C^{2}=\frac{1}{r}\left[2(a r+b) u_{\theta}+c\right] W^{1}+\frac{1}{r}(a r+b) W^{3}, \\
& C^{3}=-r \frac{\partial a(t, \theta)}{\partial \theta} W^{3}, \\
& C^{4}=(a r+b) w W^{1}+\left[(a r+b) u_{\theta}+c\right] W^{2} .
\end{aligned}
$$

\subsection{Time translation: Energy}

We first compute the conserved vector provided by the time translation symmetry $X_{1}$ from (3.1). In this case we have

$$
W^{1}=-\frac{\partial u_{\theta}}{\partial t}, \quad W^{2}=-\frac{\partial w}{\partial t}, \quad W^{3}=-\frac{\partial p}{\partial t} .
$$


Substituting these expressions into the conserved vectors $C^{i}$ given by (4.5), eliminating the derivative $\partial u_{\theta} / \partial t$ in $C^{1}$ via (2.16), using Eqs. (3.14) and removing the terms $D_{\theta}(\cdots)$ and $D_{z}(\cdots)$ from $C^{1}$ to $C^{2}$ and $C^{4}$, we write to the final form of the the conserved vector:

$$
\begin{aligned}
C^{1} & =-\frac{\partial a(t, \theta)}{\partial \theta}\left(u_{\theta}^{2}+p\right), \\
C^{2} & =\frac{1}{r}\left[\left(r \frac{\partial a(t, \theta)}{\partial t}+\frac{\partial b(t, r)}{\partial t}\right)\left(u_{\theta}^{2}+p\right)-c(t, \theta, r) \frac{\partial u_{\theta}}{\partial t}\right], \\
C^{3} & =r \frac{\partial a(t, \theta)}{\partial \theta} \frac{\partial p}{\partial t} \\
C^{4} & =\left(r \frac{\partial a(t, \theta)}{\partial t}+\frac{\partial b(t, r)}{\partial t}\right) w u_{\theta}^{2}-c(t, \theta, r) \frac{\partial w}{\partial t} .
\end{aligned}
$$

One can check by direct differentiation that the conservation law (4.3) is satisfied for the vector (4.6) in the following form:

$$
\begin{aligned}
& D_{t}\left(C^{1}\right)+D_{\theta}\left(C^{2}\right)+D_{r}\left(C^{3}\right)+D_{z}\left(C^{4}\right) \\
& =\left(r \frac{\partial a(t, \theta)}{\partial t}+\frac{\partial b(t, r)}{\partial t}\right)\left(\frac{\partial u_{\pi}}{\partial t}+\frac{u_{\theta}}{r} \frac{\partial u_{\theta}}{\partial \theta}+w \frac{\partial u_{\theta}}{\partial z}+\frac{1}{r} \frac{\partial p}{\partial \theta}\right) \\
& +r \frac{\partial a(t, \theta)}{\partial \theta}\left(\frac{\partial p}{\partial r}-\frac{u_{\theta}^{2}}{r}-f u_{\theta}\right)+\left(u_{\theta}-c \frac{\partial}{\partial t}\right)\left(\frac{1}{r} \frac{\partial u_{\theta}}{\partial \theta}+\frac{\partial w}{\partial z}\right) .
\end{aligned}
$$

The integral form of the conservation law with the vector (4.6) gives the following energy conservation for the system (2.16)-(2.20):

$$
\frac{d}{d t} \int_{R^{3}} \frac{\partial a(t, \theta)}{\partial \theta}\left(u_{\theta}^{2}+p\right) d \theta d r d z=0
$$

\subsection{Rotation: Angular momentum}

For the horizontal rotation, i.e. to the $\theta$-translation symmetry $X_{2}$ from (3.1) we have

$$
W^{1}=-\frac{\partial u_{\theta}}{\partial \theta}, \quad W^{2}=-\frac{\partial w}{\partial \theta}, \quad W^{3}=-\frac{\partial p}{\partial \theta} .
$$

Substituting these expressions in the formula (4.5) and making simplifications as above by removing the term of the form $D_{z}(\cdots)$ from $C^{2}$ to $C^{4}$, we arrive at the following conserved vector:

$$
\begin{aligned}
C^{1} & =r u_{\theta} \frac{\partial a(t, \theta)}{\partial \theta}, \\
C^{2} & =-\frac{c}{r} \frac{\partial u_{\theta}}{\partial \theta}-\left(r \frac{\partial a(t, \theta)}{\partial t}+\frac{\partial b(t, r)}{\partial t}\right) u, \\
C^{3} & =r \frac{\partial a(t, \theta)}{\partial \theta} \frac{\partial p}{\partial \theta}, \\
C^{4} & =r u_{\theta} w \frac{\partial a(t, \theta)}{\partial \theta}-c \frac{\partial w}{\partial \theta}
\end{aligned}
$$

The vector (4.9) satisfies the conservation law (4.3) in the following form:

$$
\begin{aligned}
& D_{t}\left(C^{1}\right)+D_{\theta}\left(C^{2}\right)+D_{r}\left(C^{3}\right)+D_{z}\left(C^{4}\right) \\
& =r \frac{\partial a(t, \theta)}{\partial \theta}\left(\frac{\partial u_{\theta}}{\partial t}+\frac{u_{\theta}}{r} \frac{\partial u_{\theta}}{\partial \theta}+w \frac{\partial u_{\theta}}{\partial z}+\frac{1}{r} \frac{\partial p}{\partial \theta}\right) \\
& +r \frac{\partial a(t, \theta)}{\partial \theta} \frac{\partial}{\partial \theta}\left(\frac{\partial p}{\partial r}-\frac{u_{\theta}^{2}}{r}-f u_{\theta}\right)+\left(r u_{\theta} \frac{\partial a(t, \theta)}{\partial \theta}-c \frac{\partial}{\partial \theta}\right)\left(\frac{1}{r} \frac{\partial u_{\theta}}{\partial \theta}+\frac{\partial w}{\partial z}\right) .
\end{aligned}
$$


Writing the integral form of the conservation law with the vector (4.9), we obtain the following law of conservation of the angular momentum for the system (2.16)-(2.20):

$$
\frac{d}{d t} \int_{R^{3}} \frac{\partial a(t, \theta)}{\partial \theta} r u_{\theta} d \theta d r d z=0
$$

\subsection{Conserved vector associated with scaling transformation}

For the scaling transformation generator $X_{4}$ from (3.1) we have

$$
\begin{aligned}
& W^{1}=u_{\theta}-r \frac{\partial u_{\theta}}{\partial r}-z \frac{\partial u_{\theta}}{\partial r}, \\
& W^{2}=w-r \frac{\partial w}{\partial r}-z \frac{\partial w}{\partial r}, \\
& W^{3}=2 p-r \frac{\partial p}{\partial r}-z \frac{\partial p}{\partial r}
\end{aligned}
$$

Substituting these expressions in the formula (4.5) and making simplifications as above we arrive at the following conserved vector:

$$
\begin{aligned}
& C^{1}=\left(4 a r+3 b+r b_{r}\right) u_{\theta} \\
& C^{2}=\frac{1}{r}\left(4 a r+3 b+r b_{r}\right)\left(u_{\theta}^{2}+p\right)+\left(\frac{2 c}{r}+c_{r}\right) u_{\theta}, \\
& C^{3}=-4 r p \frac{\partial a(t, \theta)}{\partial \theta}-c \frac{\partial u_{\theta}}{\partial \theta} \\
& C^{4}=\left(4 a r+3 b+r b_{r}\right) u_{\theta} w+c\left(w-r \frac{\partial w}{\partial r}\right)
\end{aligned}
$$

The vector (4.12) satisfies the conservation law (4.3) in the following form:

$$
\begin{aligned}
& D_{t}\left(C^{1}\right)+D_{\theta}\left(C^{2}\right)+D_{r}\left(C^{3}\right)+D_{z}\left(C^{4}\right)=c\left(\frac{2}{r}-\frac{\partial}{\partial r}\right)\left(\frac{\partial u_{\theta}}{\partial \theta}+r \frac{\partial w}{\partial z}\right) \\
& +\left(4 a r+3 b+r b_{r}\right)\left[\frac{\partial u_{\theta}}{\partial t}+\frac{u_{\theta}}{r} \frac{\partial u_{\theta}}{\partial \theta}+w \frac{\partial u_{\theta}}{\partial z}+\frac{1}{r} \frac{\partial p}{\partial \theta}+u_{\theta}\left(\frac{1}{r} \frac{\partial u_{\theta}}{\partial \theta}+\frac{\partial w}{\partial z}\right)\right] .
\end{aligned}
$$

The corresponding integral conservation law for the system (2.16)-(2.20) is written:

$$
\frac{d}{d t} \int_{R^{3}}\left[4 a(t, \theta) r+3 b(t, r)+r \frac{\partial b(t, r)}{\partial r}\right] u_{\theta} d \theta d r d z=0 .
$$

\section{Conclusion}

We have derived the nonlinear conservation laws corresponding to the three-dimensional Boussinesq model describing internal gravity wave p[ropagation in a cylinrical bassin obeying the Kelvin's hypothesis on the zero radial component of the velocity vector throughout the domain. We have shown that the symmetries (3.1) provide three infinite sets of nontrivial integral conservation laws, (4.8), (4.11) and (4.14), depending on three arbitrary functions, namely $a(t, \theta), b(t, r)$ and an arbitrary function involved in $c(t, \theta, r)$. Particularly, we observe that the component $C^{1}$ in the energy conservation law involves only the rotational part of the velocity vector. 
We single out physically useless conservation laws by the following definition ([13]): The conservation law is said to be trivial if its density $C^{1}$ evaluated on the solutions of the equations in question is the divergence,

$$
C^{1}=D_{x}\left(h^{1}\right)+D_{y}\left(h^{2}\right)+\ldots
$$

The conserved vector (4.5) associated with the $z$-translation symmetry $X_{3}$ vanishes on the solution manifold of the system (2.16) - (2.20). Hence, according to the above definition (5.1), it is a trivial conserved vector. Moreover, the symmetries $X_{\varphi}$ and $X_{\psi}$ also provide trivial conserved vectors whereas the symmetry $X_{5}$ from (3.1) gives a conserved vector with the vanishing density $C^{1}$.

Similar derivation of conservation laws were also obtained in our earlier work in Ref. [13], where we have investigated two-dimensional model for internal gravity waves under the Boussinesq approximaiton in the Cartesian coordinaes. The analysis in [13] were found in a good agreement with the previous work in [5] in which the classical and nonlinear stability of baroclinic jets was compared to symmetric disturbances for nonhydrostatic, adiabatic, Boussinesq equations. Within the context of the Fjortoft's theorem on an energy-Casimir (or pseudoenergy) stability analysis studied in Fjortoft, [7], the conservation law (4.11) can be associated with a Hamiltonian, which differs from the energy by a Casimir invariants which constitute "hidden" conservation laws of the Eulerian representation of fluid flows. The analysis presented in this paper extends our previous work in Ref. [13] to a three-dimensional case which also involves non-Cartesian wave field. For example we note that the energy (4.8) is a "physical" energy if $a=1$. Note that the recent paper [1] has been also devoted to obtaining "physical" conservation laws for a Boussinesq-type system. We also remark that the assumption that $N$ is constant has no any bearing on the origination of the waves modeled here (see also [16]).

\section{References}

[1] A. Ali, H. Kalisch. Mechanical balance laws for Boussinesq models of surface water waves J. Nonlinear Sci. 22 (2012), 371-398.

[2] V. Andreev. O. Kaptsov. V. Pukhnachev, A. Rodionov. Applications of group theoretic methods in hydrodynamics. Novosibirsk, Nauka. (Russian). English translation by Kluwer Academic Publishers (1994).

[3] S. Balasuriya. Vanishing viscosity in the barotropic $\beta$-plane J. Math.Anal. Appl. 214, 128-150.

[4] A. Buchnev. Lie group admitted by the equations of motion of an ideal incompressible fluid. Continuum Dynamics. 7 (1971) pp. 212-214. Institute of Hydrodynamics, USSR Acad. Sci., Siberian Branch, Novosibirsk. (Russian).

[5] H. Cho, T. Shepherd, V. Vladimirov. Application of the direct Liapunov method to the problem of symmetric stability in the atmosphere. J. Atmosph. Sci. 506 (1993), 822-836.

[6] E. Dewan, R. Picard, R. O'Neil, H. Gardiner, J. Gibson. MSX satellite observations of thunderstorm-generated gravity waves in mid-wave infrared images of the upper stratosphere. Geophys. Res. Lett. 25 (1998), 939-942.

[7] R. Fjortoft. Application of integral theorems in deriving criteria of stability for laminar flows and for the baroclinic circular vortex. Geophys. Publ., 17 (6) 1950, 1-52.

[8] A. Gill. Atmosphere-Ocean Dynamics. New York, etc., Academic Press. 1983

[9] G.H. Haltiner, R.T. Williams. Numerical prediction and dynamic meteorology 1980.

[10] P.A. Hsieh.Application of modflow for oil reservoir simulation during the Deepwater Horizon crisis. Ground Water. 49 (3), (2011), 319-323.

[11] N.H. Ibragimov. Nonlinear self-adjointness in constructing conservation laws. Archives of ALGA 7(8) (2010-2011), 1-99.

[12] N.H. Ibragimov. Nonlinear self-adjointness in constructing conservation laws. arXiv: 1109.1728v1[math-ph], (2011), 1-104.

[13] N.H. Ibragimov, R.N. Ibragimov. Rotationally symmetric internal gravity waves. J. Non-Linear Mech. (2011), doi:10.1017/j.ijnonlinmec.2011.08.011.

[14] R.N. Ibragimov, N. Yilmaz, A.S. Bakhtiyarov. Experimental mixing parameterization due to multiphase fluid-structure interaction. Mechanics Research Communications, 38 (2011), 261-266.

[15] D. Nethery, D.Shankar. Vertical propagation of baroclinic Kelvin waves along the west coast of India. J. Earth. Syst. Sci. 1164 (2007), 331-339.

[16] H. Kalisch, N.T. Nguyen. On the stability of internal waves. J. Phys. A. 43 (2010), 495205.

[17] R.D. Romea, J.S. Allen. On vertically propagating coastal Kelvin waves at low latitudes. J. Phys. Oceanogr. 13 (1983) $1,241-1,254$.

[18] D.T. Shindell, G.A. Schmidt. Southern Hemisphere climate response to ozone changes and greenhouse gas increases Res. Lett. 31 (2004), L18209. 
N. H. Ibragimov, R. N. IbragimovNonlinear Conservation Laws for Internal Gravity Waves in a Rotating Cylindrical Wave Field

[19] C. Staquet, J. Sommeria. Internal Gravity Waves: From instabilities to turbulence. Annu. Rev. Fluid Mech. 34 (2002), 559-593.

[20] R. Szoeke, R.M. Samelson. The duality between the Boussinesq and non-Boussinesq hydrostatic equations of motion. J. Phys. Oceanogr. 32 (2002), 2194-2203. 